\title{
Neuroprotection induced by post-conditioning following ischemia/reperfusion in mice is associated with altered microRNA expression
}

\author{
WEI MIAO ${ }^{1}$, TIAN-HAO BAO ${ }^{2}$, JIAN-HONG HAN $^{1}$, MEI YIN $^{1}$, JIE ZHANG ${ }^{1}$, YONG YAN $^{1}$ and YU-HONG ZHU $^{1}$ \\ ${ }^{1}$ Department of Neurology, Second Affiliated Hospital of Kunming Medical University, Kunming, Yunnan 650031; \\ ${ }^{2}$ Department of Geratology, Psychiatric Hospital of Yunnan Kunming, Yunnan 650224, P.R. China
}

Received September 17, 2015; Accepted June 24, 2016

DOI: $10.3892 / \mathrm{mmr} .2016 .5576$

\begin{abstract}
Ischemic preconditioning and ischemic postconditioning (IPostC) represent promising strategies to reduce ischemia-reperfusion (I/R) injury and attenuate the lethal ischemic damage following stroke. However, the mechanism underlying this attenuation remains to be elucidated. It was hypothesized that alterations in microRNA (miRNA) expression in the cerebral cortex and hippocampus of mice following $\mathrm{I} / \mathrm{R}$ is associated with the functional improvement induced by IPostC. Behavioral changes were assessed in a mouse model of $\mathrm{I} / \mathrm{R}$ in the absence or presence of IPostC, followed by microarray analyses to investigate the expressional alterations of miRNAs in the cerebral cortex and hippocampus of mice. The results of the present study revealed that IPostC abrogated the neurological impairment and hippocampus-associated cognitive deficits induced by I/R, and upregulated or downregulated the expression levels of numerous miRNAs. Furthermore, the upregulation of miR-19a, and the downregulation of miR-1, let-7f and miR-124 expression levels following IPostC was confirmed utilizing reverse transcription-quantitative polymerase chain reaction. The results of the present study demonstrated that alterations in miRNA expression in the cerebral cortex and hippocampus of mice following I/R was associated with the neuroprotection induced by IPostC.
\end{abstract}

\section{Introduction}

Ischemic brain injury is the underlying pathophysiology of various common diseases, including traumatic injury and stroke, which is the second leading cause of mortality

Correspondence to: Professor Yu-Hong Zhu, Department of Neurology, Second Affiliated Hospital of Kunming Medical University, 374 Dianmian Road, Kunming, Yunnan 650031, P.R. China

E-mail: zhuyuhong2014@126.com

Key words: ischemia-reperfusion, ischemic postconditioning, miRNA, cognitive functions worldwide and a primary cause of disability (1). Rapid revascularization of the occluded vessels and early reperfusion are recommended to limit cerebral ischemic damage. However, ischemia-reperfusion (I/R) injury may occur (2), causing postischemic tissue damage (3). Efforts have been made to alter the patterns of reperfusion, thus alleviating I/R injury $(3,4)$. However, to date, effective and safe methods to reduce ischemic brain injury remain to be established.

Ischemic postconditioning (IPostC), an emerging concept for stroke treatment, refers to a series of rapid intermittent blood flow interruptions early following reperfusion that alters the blood flow hydrodynamics $(4,5)$. Previous studies have demonstrated that IPostC reduced infarct size, diminished necrosis and apoptosis, improved vascular endothelial dysfunction and restored neurological deficits following stroke, in numerous human organs and various animal models (5-7). IPostC compares well with ischemic preconditioning (4) and has demonstrated comparable protective effects $(5,8)$. Previous studies have partially revealed the protective mechanisms underlying IPostC, which include: i) Attenuation of free radical generation $(2,5)$; ii) inhibition of neutrophil infiltration and attenuation of proinflammatory cytokine and adhesion molecule expression in ischemic brain (5); and iii) promotion of neuronal survival molecular pathways (9) and inactivation of apoptotic cell signaling pathways $(5,10)$. However, the majority of these studies were descriptive and lacked insight into the underlying molecular mechanisms of IPostC.

MicroRNAs (miRNAs), a novel class of noncoding RNAs, are important endogenous regulators that post-transcriptionally modulate the expression of target mRNAs via degradation or translational inhibition (11). miRNAs are critical for the maintenance of healthy cellular function. Quantifying miRNA expression levels and predicting their function as regulators of single targets and complex networks requires a combined approach of bioinformatics, molecular and systems biology. Previous studies have suggested the involvement of miRNAs in the regulation of I/R injury $(12,13)$. However, alterations in miRNAs induced by cerebral IPostC in an I/R mouse model remain to be fully elucidated.

The present study examined alterations in miRNA expression levels in the cerebral cortex and hippocampus in an $\mathrm{I} / \mathrm{R}$ mouse model following IPostC treatment using 
microarray analyses. Mice were subjected to I/R in the presence or absence of IPostC. Subsequently, their neurological functional impairment, and spatial learning and memory retention abilities were assessed. The cerebral cortex and hippocampus were then collected and miRNA analysis was performed. The results of miRNA array were confirmed using reverse transcription-quantitative polymerase chain reaction (RT-qPCR).

\section{Materials and methods}

Animal grouping and experimental design. Animals were randomly divided into two groups: i) I/R group $(n=16)$, in which animals underwent $45 \mathrm{~min}$ ischemia followed by $72 \mathrm{~h}$ reperfusion; ii) IPostC group ( $\mathrm{n}=17)$, in which I/ $\mathrm{R}$ was followed by three cycles of $15 \mathrm{sec}$ occlusion/30 sec release started at 2 min subsequent to reperfusion (IP15/30) (14).

Focal cerebral ischemia and IPostC. All investigations conformed to the Guide for the Care and Use of Laboratory Animals published by the National Institutes of Health (NIH Publication No. 85-23, revised 1996; Bethesda, MD, USA). The study was approved by the ethics committee of the Second Affiliated Hospital of Kunming Medical University (Kunming, China; permit no. ku-sah-2015004). A total of 36 male C57BL/6J mice (age, 4-4.5 months; weight, 22-25 g) were purchased from Kunming Medical University (Kunming, China). Animals were maintained on a standard diet and water accessed ad libitum, and housed at $20-25^{\circ} \mathrm{C}$ under a 12-h light/dark cycle. Efforts were made to minimize animal numbers and suffering.

Anesthesia was induced by $5 \%$ isoflurane (Sigma-Aldrich China, Inc., Shanghai, China) and maintained with 1-2\% isoflurane during surgery. Focal ischemia was generated as described previously $(9,14)$. Body core temperature was monitored with a rectal probe and maintained at $37 \pm 0.5^{\circ} \mathrm{C}$ using a heat mat. Briefly, under the operating microscope, the left common carotid artery (CCA) and external carotid artery (ECA) were exposed via a ventral midline neck incision, and were ligated proximally. A 6-0 silicon-coated nylon suture with a $0.23 \mathrm{~mm}$ tip diameter (Doccol Corporation, Sharon, MA, USA) was inserted through the arteriotomy in the CCA just below the carotid bifurcation $8 \pm 0.5 \mathrm{~mm}$ until a mild resistance was felt. The inserted suture was held in place with a 5-0 black silk suture (Beijing Cinontech Co., Ltd., Beijing, China) at the proximal CCA bifurcation. The suture was removed $45 \mathrm{~min}$ later to allow reperfusion in the ischemic control group. In the IPostC group, the suture was removed 2-3 $\mathrm{mm}$ and reinserted repeatedly as described previously (14). Following surgery, animals were returned to their cages. Three mice died following surgery (two in the I/R group and one in the IPostC group). Therefore, subsequent analyses were performed on 16 mice in the I/R group and 17 mice in the IPostC group.

Neurological score evaluation. Neurological scores were assessed at 1,2 and 3 days post-operation (dpo). A 28 point scale of focal neurological scores (FNS) was employed as described previously (15), which comprised the following seven tests, all of which were scored from 0 to 4 : i) Body symmetry, ii) gait, iii) climbing, iv) circling behavior, v) frontal limb symmetry, vi) compulsory circling, and vii) whisker response. Scores for each category were added up, giving a total score for each animal of 0-28.

Behavioral evaluations. A total of 14 days following surgery, the learning and memory impairment of mice were assessed. A Morris water maze (MWM) was used to examine spatial learning by training mice to locate an underwater platform using visual information, as described previously (16). The test was conducted three times per day each day from 15 to $20 \mathrm{dpo}$. The time required to find the hidden platform (escape latency; time limit, $90 \mathrm{sec}$ ) was recorded by an observer blinded to the treatment group and tracked using TopScan software version 3.1 (Clever Sys Inc., Reston, VA, USA). A $90 \mathrm{sec}$ probe trial was performed 1 day subsequent to the final learning trial to assess memory. The platform was removed and the percentage of time spent in the quadrant where it was previously located was recorded.

miRNA microarray analysis. The miRCURY LNA ${ }^{\mathrm{TM}}$ Array (7th generation; version 18.0; Exiqon A/S, Vedbaek, Denmark) contains 3,100 capture probes, covering all human, mouse and rat miRNAs annotated in miRBase version 18.0 (www.mirbase. org/), and all viral miRNAs associated with these species.

Mice $(n=3)$ were randomly selected from each group 20 days following surgery, subsequent to the MWM test, sacrificed by cervical dislocation and decapitated. Ischemic ipsilateral cortex and hippocampus were removed within $60 \mathrm{sec}$ and frozen in $-70^{\circ} \mathrm{C}$ isopentane until further analysis. Following careful rinsing in chilled phosphate-buffered saline, tissues from the three mice in each group were pooled and homogenized on ice using TRIzol ${ }^{\circledR}$ (Invitrogen; Thermo Fisher Scientific, Inc., Waltham, MA, USA), and total RNA was extracted using TRIzol ${ }^{\circledR}$ and a miRNeasy Mini kit (Qiagen, Inc., Valencia, CA, USA) according to manufacturer's instructions. RNA quality and quantity were examined using a Nanodrop spectrophotometer (ND-1000; NanoDrop Technologies; Thermo Fisher Scientific, Inc.) and RNA integrity was assessed by gel electrophoresis.

Following RNA isolation from the samples, the miRCURY Hy3/Hy5 $5^{\text {TM }}$ Power Labeling kit (Exiqon A/S) was used according to the manufacturer's instructions. The $\mathrm{Hy} 3^{\mathrm{TM}}$-labeled samples were hybridized on the miRCURY LNA $^{\mathrm{TM}}$ Array according to the manufacturer's instructions. The slides were then washed repeatedly with the Wash Buffer kit (Exiqon A/S) and centrifuged for $5 \mathrm{~min}$ at $80 \mathrm{x} \mathrm{g}$, $20^{\circ} \mathrm{C}$ ). Slides were scanned with the Axon GenePix ${ }^{\circledR} 4000 \mathrm{~B}$ microarray scanner (Molecular Devices, LLC, Sunnyvale, CA, USA).

Scanned images were then imported into GenePix ${ }^{\circledR}$ Pro software version 6.0 (Molecular Devices, LLC) for grid alignment and data extraction. Replicated miRNAs were averaged and miRNAs with intensities $\geq 30$ in all samples were chosen for normalization. Expressed data were normalized using the Median normalization (17). Subsequent to normalization, differentially expressed (DE) miRNAs were identified through Fold Change filtering [only normalized intensity ratios $>2.0$ or $<0.5$ (fold-changes $\geq 2.0$ ) were defined as significantly altered miRNAs]. Hierarchical clustering was 
performed using MultiExperiment Viewer software, version 4.0 (www.tm4.org/mev.html) for classification analysis.

Expressional analysis of miRNA. RT-qPCR was performed to measure miRNA expression levels. The remaining mice (13 in the $\mathrm{I} / \mathrm{R}$ and 14 in the IPostC groups) were sacrificed for miRNA analysis immediately following MWM assessment. Complementary DNA (cDNA) was synthesized from total RNA, and qPCR performed using gene-specific primers and the TaqMan ${ }^{\circledR}$ MicroRNA assay kit (Applied Biosystems; Thermo Fisher Scientific, Inc.), according to the manufacturer's instructions. The $10 \mu \mathrm{l}$ PCR reaction contained $0.67 \mu \mathrm{l}$ cDNA, $4 \mu 11 \mathrm{X}$ TaqMan Universal PCR master mix and $1 \mu \mathrm{l}$ primer and probe mix. qPCR was performed using an Applied Biosystems 7300 Sequence Detection system (Applied Biosystems; Thermo Fisher Scientific, Inc.). Samples were normalized to snoRNA202 (18). The threshold cycle was defined as the fractional cycle number at which the fluorescence exceeded the fixed threshold (19). The relative expression of genes was determined using the $\Delta \Delta \mathrm{Cq}$ method (20).

Statistical analysis. Data are expressed as the mean \pm standard error. All statistical analyses were performed in SPSS version 14.0 (SPSS, Inc., Chicago, IL, USA). FNS were analyzed using the Kruskal-Wallis test followed by the Mann-Whitney U-test with Bonferroni correction. Post-hoc independent samples $t$-tests were used to assess significant differences between groups. $\mathrm{P}<0.05$ was considered to indicate a statistically significant difference.

\section{Results}

Behavioral evaluation. Increased FNS indicated greater impairment (15). IPostC significantly decreased neurological scores at $1(\mathrm{P}=0.01), 2(\mathrm{P}=0.0024)$ and $3(\mathrm{P}<0.001)$ dpo compared with the $\mathrm{I} / \mathrm{R}$ only group (Fig. 1 ). In addition, in the IPostC group but not in the I/R only group, FNS was significantly decreased at 2 and 3 dpo compared with 1 dpo.

Spatial orientation alteration. All mice subjected to I/R injury demonstrated significantly decreased escape latency from 16 dpo (Fig. 2). However, in the IPostC group, mice discovered the platform more rapidly than I/R alone mice, from $16 \mathrm{dpo}$ to $20 \mathrm{dpo}$. In the probe test, IPostC mice spent a significantly increased percentage of time in the target quadrant on 21 dpo compared with I/R group mice, indicating that IPostC attenuated I/R-induced memory impairment.

IPostC resulted in DE miRNAs in cerebral cortex and hippocampus of I/R mice. To identify DE miRNAs induced by IPostC, a Fold Change filtering was performed. The miRNA expression profile heat map of these groups was generated by hierarchical clustering. The color gradient of the heat map represents the log of relative to mean miRNA expression, with red indicating overexpression and green underexpression. Hierarchical cluster analysis of these miRNAs identified DE miRNAs in cerebral cortex (Fig. 3) and hippocampus (Fig. 4) induced by IPostC.

In the cerebral cortex, 39 miRNAs were DE in $\mathrm{I} / \mathrm{R}$ and IPostC mice, of which 21 were upregulated, and 18 were

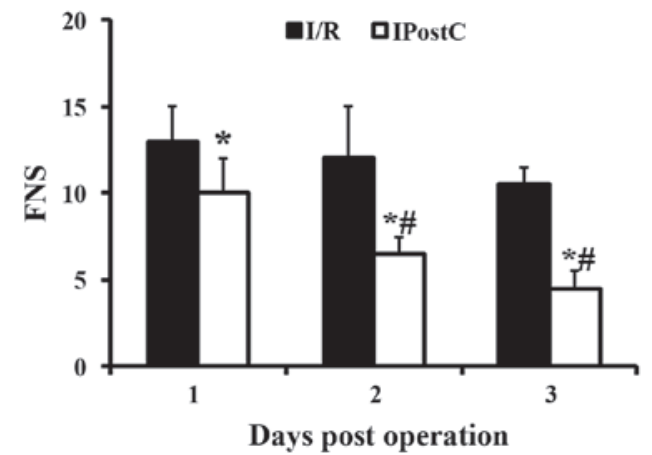

Figure 1. FNS are decreased by IPostC. FNS were calculated at 1,2 and 3 dpo. IPostC significantly decreased neurological scores at 1, 2 and 3 dpo compared with the I/R only group. In addition, in the IPostC group but not in the I/R only group, FNS significantly decreased over time. Data are expressed as the mean \pm standard error. ${ }^{*} \mathrm{P}<0.05$ vs. I/R alone on the corresponding day; ${ }^{\#} \mathrm{P}<0.05$ vs. IPostC group $1 \mathrm{dpo}$. FNS, focal neurological score; I/R, ischemia-reperfusion; IPostC, ischemic postconditioning; dpo, days post operation.

A

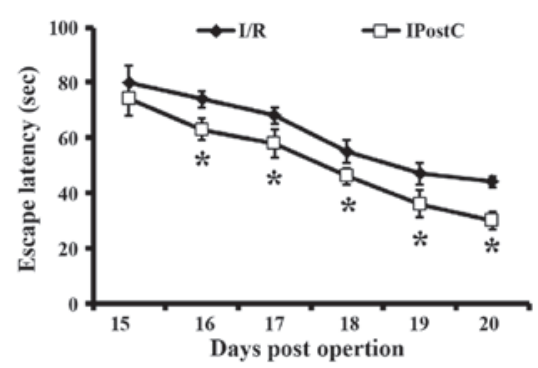

B

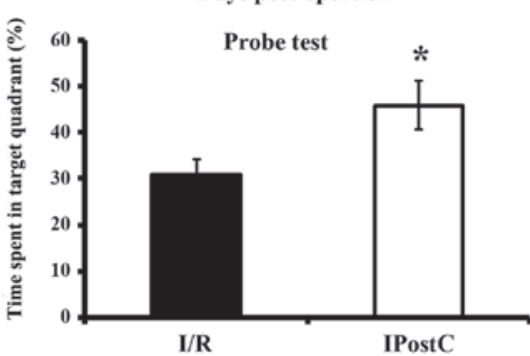

Figure 2. Behavioral changes evaluated by the Morris water maze test. (A) The duration of time required for mice to discover the submerged platform (the escape latency) was recorded three times per day for six consecutive days. IPostC group mice reached the platform significantly more rapidly than I/R alone mice from 16 dpo onwards. (B) The probe test was performed at $21 \mathrm{dpo}$, following removal of the platform. IPostC group mice spent a significantly increased percentage of time in the quadrant where the platform was previously located. Data are expressed as the mean \pm standard error. ${ }^{*} \mathrm{P}<0.05$ vs. I/R alone. I/R, ischemia-reperfusion; IPostC, ischemic postconditioning; dpo, days post operation.

downregulated (Table I and Fig. 3). In addition, as presented in Table II and Fig. 4, IPostC induced DE miRNAs in the hippocampus. A total of 27 miRNAs were DE in I/R and IPostC groups, of which 13 were upregulated, and 14 were downregulated. The four miRNAs (miR-1, let-7f, miR-19a and miR-124) that were DE in cerebral cortex and hippocampus were selected for further analysis.

Quantitative analysis of miRNAs. The expression levels of miR-1, let-7f, miR-19a and miR-124 were evaluated using RT-qPCR. In the cerebral cortex, miR-19a expression levels 
Table I. Differentially expressed miRNAs in the cerebral cortex of I/R and IPostC mice.

\begin{tabular}{|c|c|c|}
\hline miRNA ID & $\begin{array}{c}\text { Fold-change } \\
\text { (IPostC vs. I/R) }\end{array}$ & P-value \\
\hline mmu-miR-350-3p & 2.97 & 0.005271 \\
\hline mmu-miR-138-1-3p & 2.37 & 0.007454 \\
\hline mmu-miR-18b-5p & 2.57 & 0.005162 \\
\hline mmu-miR-448-3p & 4.68 & 0.000613 \\
\hline mmu-miR-465a-5p & 4.81 & 0.000516 \\
\hline mmu-miR-21 & 8.15 & 0.000361 \\
\hline mmu-miR-668-3p & 6.71 & 0.000444 \\
\hline mmu-miR-18a-5p & 10.45 & 0.000135 \\
\hline mmu-miR-302a-3p & 4.98 & 0.000513 \\
\hline mmu-miR-19a & 6.12 & 0.000423 \\
\hline mmu-miR-3084-3p & 2.11 & 0.008135 \\
\hline mmu-miR-17-5p & 2.04 & 0.008396 \\
\hline mmu-miR-3966 & 8.68 & 0.000323 \\
\hline mmu-miR-1298-5p & 3.04 & 0.005049 \\
\hline mmu-miR-466d-3p & 3.37 & 0.004954 \\
\hline mmu-miR-669m-3p & 3.76 & 0.004239 \\
\hline mmu-miR-3084-5p & 2.02 & 0.009231 \\
\hline mmu-miR-145 & 2.94 & 0.005365 \\
\hline mmu-miR-3099-5p & 38.63 & 0.000000 \\
\hline mmu-miR-3081-3p & 2.03 & 0.008132 \\
\hline mmu-miR-128-2-5p & 2.14 & 0.007942 \\
\hline mcmv-miR-m01-2-5p & 0.37 & 0.002235 \\
\hline mmu-miR-1 & 0.45 & 0.007359 \\
\hline mmu-miR-200c-3p & 0.04 & 0.000321 \\
\hline mmu-miR-16 & 0.24 & 0.001132 \\
\hline mmu-miR-374c-3p & 0.35 & 0.002924 \\
\hline mmu-miR-191 & 0.13 & 0.000923 \\
\hline mmu-miR-3473a & 0.43 & 0.007549 \\
\hline mmu-miR-3072-3p & 0.42 & 0.007854 \\
\hline mmu-let-7f & 0.33 & 0.002139 \\
\hline mmu-miR-5616-3p & 0.28 & 0.001831 \\
\hline mmu-miR-141-5p & 0.19 & 0.000965 \\
\hline mmu-miR-145 & 0.42 & 0.007715 \\
\hline mmu-miR-1933-3p & 0.19 & 0.000932 \\
\hline mmu-miR-352 & 0.32 & 0.002356 \\
\hline mmu-miR-124 & 0.32 & 0.002646 \\
\hline mmu-miR-138 & 0.35 & 0.002524 \\
\hline mmu-miR-693-5p & 0.25 & 0.001692 \\
\hline mmu-miR-106b-5p & 0.40 & 0.000364 \\
\hline
\end{tabular}

miRNA, microRNA.

were significantly increased $(\mathrm{P}<0.001)$, while miR-1 $(\mathrm{P}=0.007)$, let-7f $(\mathrm{P}=0.002)$ and miR-124 $(\mathrm{P}=0.003)$ expression levels were significantly decreased, in the IPostC compared with the I/R group (Fig. 5A). Similar observations were made in the hippocampus of IPostC-treated mice, which demonstrated an upregulation of miR-19a expression levels $(\mathrm{P}<0.001)$ and downregulation of miR-1 $(\mathrm{P}=0.002)$, let-7f $(\mathrm{P}=0.001)$ and
Table II. Differentially expressed miRNAs in the hippocampus of I/R and IPostC mice.

\begin{tabular}{lcc}
\hline & $\begin{array}{c}\text { Fold-change } \\
\text { (IPostC vs. I/R) }\end{array}$ & P-value \\
\hline miRNA ID & 4.25 & 0.000571 \\
mmu-miR-152-3p & 6.83 & 0.000354 \\
mmu-miR-27a-3p & 3.20 & 0.004162 \\
mmu-miR-374b-5p/ & 7.24 & 0.000213 \\
mmu-miR-374c-5p & & \\
mmu-miR-181c-5p & 4.89 & 0.000513 \\
mmu-miR-19a & 7.80 & 0.000361 \\
mmu-miR-101a-3p & 8.28 & 0.000244 \\
mmu-miR-431-5p & 4.76 & 0.000535 \\
mmu-miR-187-3p & 3.82 & 0.001813 \\
mmu-miR-301b-3p & 7.52 & 0.000123 \\
mmu-miR-20b-5p & 3.42 & 0.002135 \\
mmu-miR-132-3p & 6.37 & 0.003826 \\
mmu-miR-666-3p & 22.44 & 0.000012 \\
mmu-miR-450a-1-3p & 0.39 & 0.002615 \\
mghv-miR-M1-2-5p & 0.21 & 0.001259 \\
mmu-miR-1 & 0.34 & 0.002211 \\
mmu-miR-21 & 0.48 & 0.011932 \\
mmu-miR-34a & 0.49 & 0.009924 \\
mmu-let-7f & 0.29 & 0.001023 \\
mmu-miR-1843b-3p & 0.38 & 0.002549 \\
mmu-miR-124 & 0.04 & 0.000554 \\
mmu-miR-291a-5p & 0.14 & 0.00001356 \\
mmu-miR-542-5p & 0.04 & \\
mmu-miR-26a & 0.21 & \\
mmu-miR-18 & 0.22 & \\
mmu-miR-290 & 0.23 & \\
mmu-miR-466f-5p & & \\
\hline miRNA, microRNA. & & \\
\hline & & \\
\hline
\end{tabular}

miR-124 ( $\mathrm{P}=0.001)$ expression levels, compared with the $\mathrm{I} / \mathrm{R}$ group (Fig. 5B).

\section{Discussion}

The present study revealed that IPostC, consisting of three cycles of $15 \mathrm{sec}$ occlusion/30 $\mathrm{sec}$ release started $2 \mathrm{~min}$ following reperfusion, attenuated neurological impairment and hippocampus-associated cognitive deficits induced by I/R injury. In addition, it was demonstrated that IPostC induced alterations in miRNAs expression levels in the cerebral cortex and hippocampus following I/R. In particular, miR-1, let-7f, miR-19a and miR-124 expression levels were significantly altered by IPostC. These results indicate that modulation of miRNA expression by IPostC may contribute to the cognitive improvement of these mice following I/R injury.

FNS evaluation and MWM test results revealed that I/R produced significant and irreversible neurological deficits and long-term impairment in the cognitive abilities of mice, 


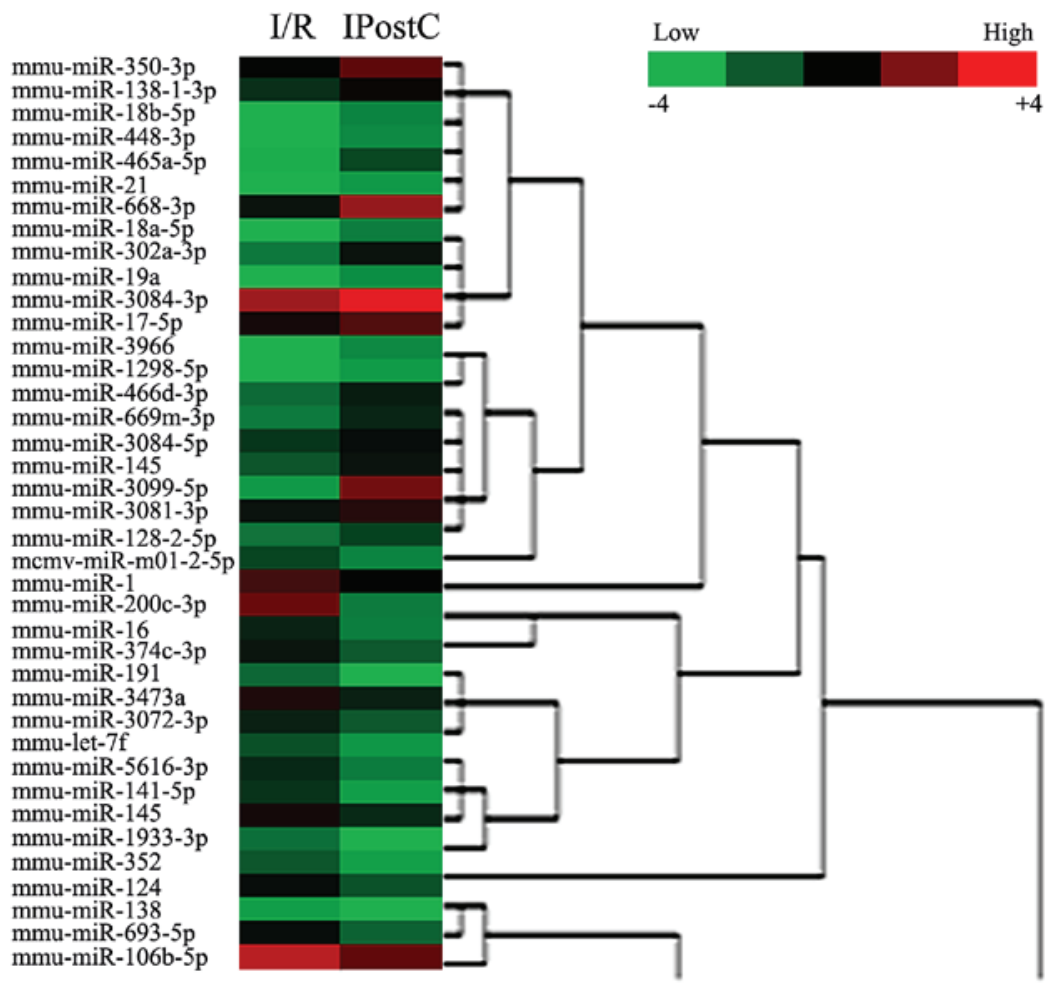

Figure 3. Heat map and hierarchical clustering dendrogram of significant DE miRNAs in cerebral cortex induced by IPostC. The heat map presents the upregulated and downregulated significant DE miRNAs, while the dendrogram reveals the hierarchical clustering of the miRNAs $(\mathrm{P}<0.05)$. The red and green color scale represents high and low expression, respectively. miRNA, microRNA; I/R, ischemia-reperfusion; IPostC, ischemic postconditioning; DE, differentially expressed.

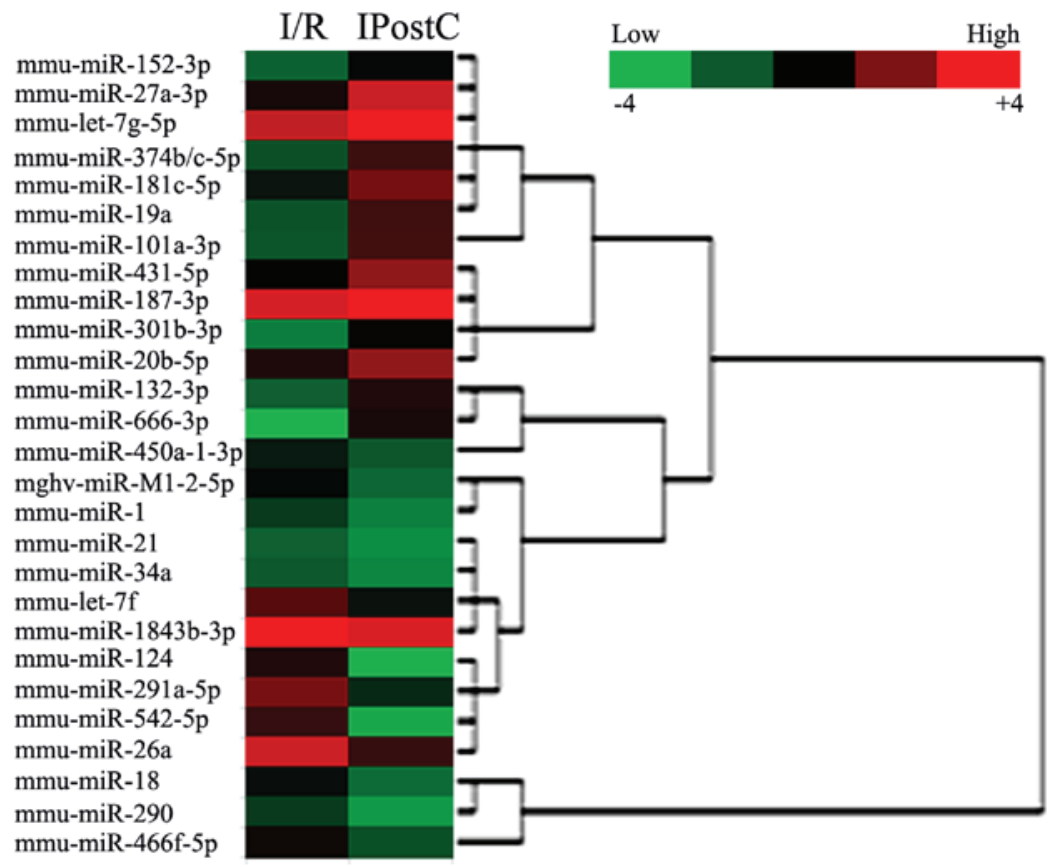

Figure 4. Heat map and hierarchical clustering dendrogram of significant DE miRNAs in hippocampus induced by IPostC. The heat map presents the upregulated and downregulated significant DE miRNAs, while the dendrogram reveals the hierarchical clustering of the miRNAs $(\mathrm{P}<0.05)$. The red and green color scale represents high and low expression, respectively. miRNA, microRNA; I/R, ischemia-reperfusion; IPostC, ischemic postconditioning; DE, differentially expressed.

consistent with previous studies $(14,21)$. However, in the present study, treatment with IPostC attenuated neurological deficits and cognitive performance in the MWM test induced by I/R. Previous studies have revealed that IPostC ameliorates neurological deficits and inhibited brain injury following stroke $(22,23)$. It has been demonstrated that I/R destroys 
A
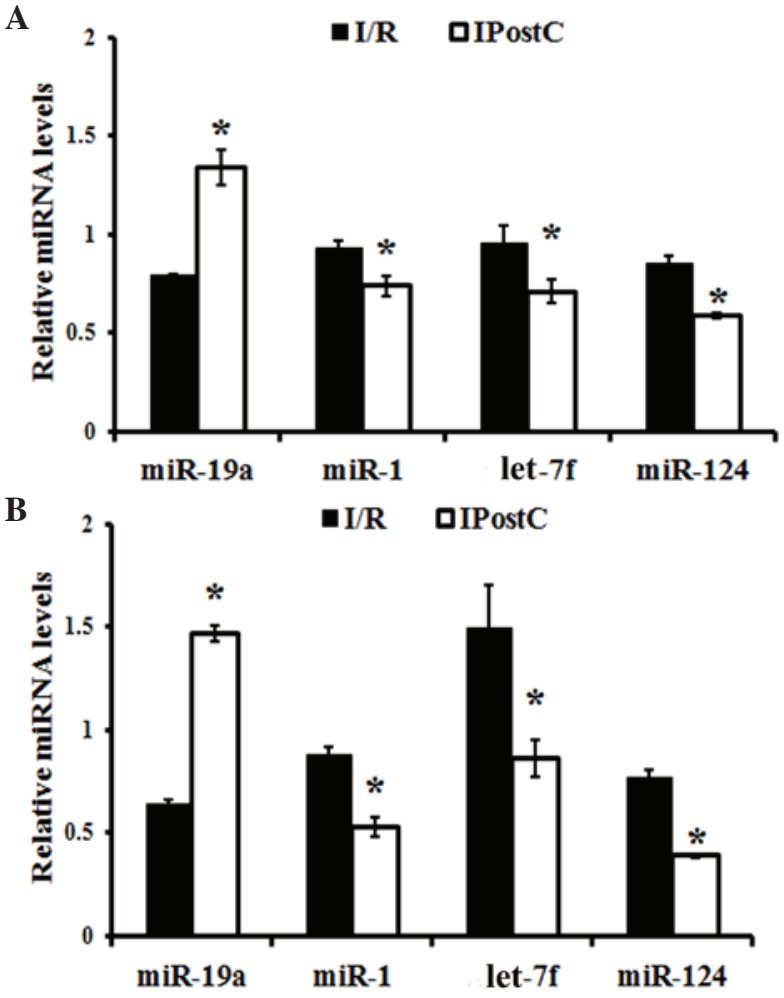

Figure 5. miRNA expression levels analyzed by reverse transcription-quantitive polymerase chain reaction. Expression levels of miR-19a, miR-1, let-7f and miR-124 were measured in (A) cerebral cortex and (B) hippocampus. miR-19a expression levels were significantly increased, while miR-1, let-7f and miR-124 expression levels were significantly decreased, in the IPostC compared with the I/R group. Data are expressed as the mean \pm standard error. "P<0.05 vs. I/R alone. miRNA, microRNA; I/R, ischemia-reperfusion; IPostC, ischemic postconditioning.

up to $85.8 \%$ of CA1 hippocampal neurons and $64.1 \%$ of parietal cortical neurons, which contribute to the cognitive impairment following injury (10). Evidence demonstrates the results of IPostC: i) Markedly reduced neuronal loss and delayed neuronal death following reperfusion; ii) significantly decreased neurological deficit scores, infarct volume and brain edema; and iii) diminished spatial learning and memory deficiency associated with cerebral ischemia $(10,12,13)$. Although it is widely accepted that IPostC increases adult hippocampal neurogenesis and enhances behavioral performance in rodents, the mechanisms underlying this process remain to be fully elucidated. An epigenetic mechanism may be involved.

It has been demonstrated that miRNAs are crucial for the maintenance of healthy cellular function. They function primarily by binding to their target mRNAs, resulting in mRNA degradation or prevention of translation $(24,25)$. Altered miRNA expression has various consequences for mRNA transcription and translation. Evidence indicates that miRNAs are involved in the regulation of I/R injury (26). A previous study demonstrated distinct expression patterns of miRNAs in stroke etiology, including atherosclerosis, hyperlipidemia, hypertension and plaque rupture (27). In addition, it has been revealed that focal ischemia significantly altered the temporal expression of numerous miRNAs, which may regulate the mRNA transcription and translation involved in stroke pathophysiology (28).
However, limited studies have investigated alterations in miRNA expression levels in the cerebral cortex and hippocampus of mice treated with IPostC following I/R injury (26). The present study revealed that there were numerous $\mathrm{DE}$ miRNAs in the cerebral cortex and hippocampus of $I / R$ and IPostC-treated mice. Hierarchical cluster analysis of miRNA profiles suggested an epigenetic mechanism may contribute to the IPostC-associated improvement in the neurological and cognitive functions of mice suffering from I/R.

The results of the present study revealed that the expression levels of miR-1, let-7f and miR-124 were downregulated in IPostC-treated mice compared with $\mathrm{I} / \mathrm{R}$ alone. A previous study has demonstrated the upregulated expression of specific miRNAs in rodent brains following I/R, suggesting miRNAs may be involved in the complex response to I/R (24). miRNA transcripts present in the blood and brain at $24 \mathrm{~h}$ following reperfusion included rno-miR-16, -23a, -191, -292-5p, -320, $-451,-494$ and let-7, while miRNAs observed in the blood and brain at $48 \mathrm{~h}$ included miR-26a, -26b, -103, -107, -150, $-185,-195,-191,-214,-320,-328,-352,-494$ and let-7 (29). Furthermore, the expression levels of miRNAs in blood have been revealed to be reproducible and diagnostic for lung cancer, colorectal cancer and diabetes (30). It has been demonstrated that following ischemia, anti-miR-1 treatment significantly reduced cortical infarct volume in adult female rats, while anti-let7 robustly reduced cortical and striatal infarcts, and preserved sensorimotor function and interhemispheric neural integration (31). Therefore, the neurological and cognitive improvement in I/R-injured mice resulting from IPostC treatment may involve miR-1 and let-7f regulation in the cerebral cortex and hippocampus. Brain-derived neurotrophic factor (BDNF) is a neurotrophin family secreted protein that regulates brain development, synaptogenesis and memory and learning (32). Evidence suggests that endogenous miR-1 and miR-10 act cooperatively as novel regulators of BDNF long and short 3'UTR isoforms (33). In the present study, IPostC reversed the upregulation of miR-1 following $I / R$, therefore the IPostC-induced cognitive improvement in I/R may involve miR-1/BDNF. However, further investigations are required to support this.

miR-124, the brain-specific miRNA involved in neural tube development, was upregulated in rats subjected to transient cerebral ischemia (34). The present study confirmed this finding, demonstrating increased miR-124 in brains from I/R, compared with IPostC, mice. This process may be associated with regeneration during the $h$ of reperfusion in the injured brain cells. Therefore, the functional improvement induced by IPostC may be associated with expressional regulation of miR-124.

It was reported that miR-19b is critical for increasing the number of oligodendroglial cells (35). The overexpression of miR-19b downregulated phosphatase and tensin homolog protein levels and induced oligodendrocyte precursor cell proliferation via activation of downstream targets of the Akt signaling pathway [phosphoinositide 3-kinase (PI3K)/Akt/mammalian target of rapamycin (mTOR)] $(35,36)$. In addition, Xie et al (22) revealed that IPostC provided long-term protection by enhancing Akt and mTOR activity during the acute post-stroke phase, which was abolished by mTOR inhibitor rapamycin administration. Therefore, the 
upregulation of miR-19b observed in the present study may activate the PI3 K/Akt/mTOR signaling pathway, accounting for the neuroprotection provided by IPostC following I/R.

In conclusion, the results of the present study demonstrate that IPostC following $\mathrm{I} / \mathrm{R}$ resulted in an improvement in neurological and cognitive function, and alterations in miRNA expression levels in the cerebral cortex and hippocampus of mice of miR-1, let-7f, miR-19a and miR-124, alone or in combination with other miRNAs, were associated with this recovery process. Alterations in miR-1, let-7f, miR-19a and miR-124 expression in the cerebral cortex and hippocampus of mice following IPostC may be involved in this improvement. However, further experiments are required to confirm this involvement and determine the potential underlying mechanisms.

\section{References}

1. Durukan A and Tatlisumak T: Preconditioning-induced ischemic tolerance: A window into endogenous gearing for cerebroprotection. Exp Transl Stroke Med 2: 2, 2010.

2. Zhao H, Sapolsky RM and Steinberg GK: Interrupting reperfusion as a stroke therapy: Ischemic postconditioning reduces infarct size after focal ischemia in rats. J Cereb Blood Flow Metab 26: 1114-1121, 2006

3. Lin XM, Zhang ZY, Wang LF, Zhang L, Liu Y, Liu XL, Yang XC, Cui $\mathrm{L}$ and Zhang L: Attenuation of tumor necrosis factor-alpha elevation and improved heart function by postconditioning for $60 \mathrm{sec}$ in patients with acute myocardial infarction. Chin Med J (Engl) 123: 1833-1839, 2010.

4. Zhao ZQ, Corvera JS, Halkos ME, Kerendi F, Wang NP, Guyton RA and Vinten-Johansen J: Inhibition of myocardial injury by ischemic postconditioning during reperfusion: Comparison with ischemic preconditioning. Am J Physiol Heart Circ Physiol 285: H579-H588, 2003.

5. Zhao H: Ischemic postconditioning as a novel avenue to protect against brain injury after stroke. J Cereb Blood Flow Metab 29 873-885, 2009

6. Zhuo C, Wang Y, Wang X, Wang Y and Chen Y: Cardioprotection by ischemic postconditioning is abolished in depressed rats: Role of Akt and signal transducer and activator of transcription-3. Mol Cell Biochem 346: 39-47, 2011.

7. Ma XJ, Yin HJ, Guo CY, Jiang YR, Wang JS and Shi DZ: Ischemic postconditioning through percutaneous transluminal coronary angioplasty in pigs: Roles of PI3K activation. Coron Artery Dis 23: 245-250, 2012.

8. Zhao $\mathrm{H}$ : The protective effects of ischemic postconditioning against stroke: From rapid to delayed and remote postconditioning. Open Drug Discov J 5: 138-147, 2011.

9. Xiong X, Gu L, Zhang H, Xu B, Zhu S and Zhao H: The protective effects of $\mathrm{T}$ cell deficiency against brain injury are ischemic model-dependent in rats. Neurochem Int 62: 265-270, 2013

10. Wang JY, Shen J, Gao Q, Ye ZG, Yang SY, Liang HW, Bruce IC, Luo BY and Xia Q: Ischemic postconditioning protects against global cerebral ischemia/reperfusion-induced injury in rats. Stroke 39: 983-990, 2008.

11. Ambros V: MicroRNA pathways in flies and worms: Growth, death, fat, stress, and timing. Cell 113: 673-676, 2003.

12. Wang X, Zhang X, Ren XP, Chen J, Liu H, Yang J, Medvedovic M, $\mathrm{Hu} \mathrm{Z}$ and Fan GC: MicroRNA-494 targeting both proapoptotic and antiapoptotic proteins protects against ischemia/reperfusion-induced cardiac injury. Circulation 122: 1308-1318, 2010.

13. Peng B, Guo QL, He ZJ, Ye Z, Yuan YJ, Wang N and Zhou J: Remote ischemic postconditioning protects the brain from global cerebral ischemia/reperfusion injury by up-regulating endothelial nitric oxide synthase through the PI3K/Akt pathway. Brain Res 1445: 92-102, 2012.

14. Joo SP, Xie W, Xiong X, Xu B and Zhao H: Ischemic postconditioning protects against focal cerebral ischemia by inhibiting brain inflammation while attenuating peripheral lymphopenia in mice. Neuroscience 243: 149-157, 2013.
15. Hill JK, Gunion-Rinker L, Kulhanek D, Lessov N, Kim S, Clark WM, Dixon MP, Nishi R, Stenzel-Poore MP and Eckenstein FP: Temporal modulation of cytokine expression following focal cerebral ischemia in mice. Brain Res 820: 45-54, 1999.

16. Loane DJ, Pocivavsek A, Moussa CE, Thompson R, Matsuoka Y, Faden AI, Rebeck GW and Burns MP: Amyloid precursor protein secretases as therapeutic targets for traumatic brain injury. Nat Med 15: 377-379, 2009.

17. Min XL, Wang TY, Cao Y, Liu J, Li JT and Wang TH: MicroRNAs: A novel promising therapeutic target for cerebral ischemia/reperfusion injury? Neural Regen Res 10: 1799-1808, 2015.

18. Bhalala OG, Pan L, Sahni V, McGuire TL, Gruner K, Tourtellotte WG and Kessler JA: MicroRNA-21 regulates astrocytic response following spinal cord injury. J Neurosci 32: 17935-17947, 2012

19. Ouchi Y, Banno Y, Shimizu Y, Ando S, Hasegawa H, Adachi K and Iwamoto $\mathrm{T}$ : Reduced adult hippocampal neurogenesis and working memory deficits in the Dgcr8-deficientmouse model of 22q11.2 deletion-associated schizophrenia can be rescued by IGF2. J Neurosci 33: 9408-9419, 2013.

20. Livak KJ and Schmittgen TD: Analysis of relative gene expression data using real-time quantitative PCR and the 2(-Delta Delta C(T)) method. Methods 25: 402-408, 2001.

21. Li H, Yin J, Li L, Deng J, Feng C and Zuo Z: Isoflurane postconditioning reduces ischemia-induced nuclear factor- $\kappa \mathrm{B}$ activation and interleukin $1 \beta$ production to provide neuroprotection in rats and mice. Neurobiol Dis 54: 216-224, 2013.

22. Xie R, Wang P, Ji X and Zhao H: Ischemic post-conditioning facilitates brain recovery after stroke by promoting Akt/mTOR activity in nude rats. J Neurochem 127: 723-732, 2013.

23. Rezazadeh H, Hoseini Kahnuee M, Roohbakhsh A, Shamsizadeh A, Rahmani MR, Bidaki R, Amin F, Kamali B, Bakhshi $\mathrm{H}$ and Allahtavakoli M: Neuroprotective consequences of postconditioning on embolic model of cerebral ischemia in rat. Iran J Basic Med Sci 16: 144-149, 2013.

24. Zacharewicz E, Lamon S and Russell AP: MicroRNAs in skeletal muscle and their regulation with exercise, ageing, and disease. Front Physiol 4: 266, 2013.

25. Humphreys DT, Westman BJ, Martin DI and Preiss T: MicroRNAs control translation initiation by inhibiting eukaryotic initiation factor 4E/cap and poly(A) tail function. Proc Natl Acad Sci USA 102: 16961-16966, 2005

26. Weiss JB, Eisenhardt SU, Stark GB, Bode C, Moser M and Grundmann S: MicroRNAs in ischemia-reperfusion injury. Am J Cardiovasc Dis 2: 237-247, 2012.

27. Rink $C$ and Khanna S: MicroRNA in ischemic stroke etiology and pathology. Physiol Genomics 43: 521-528, 2011.

28. Dharap A, Bowen K, Place R, Li LC and Vemuganti R: Transient focal ischemia induces extensive temporal changes in rat cerebral MicroRNAome. J Cereb Blood Flow Metab 29: 675-687, 2009.

29. Jeyaseelan K, Lim KY and Armugam A: MicroRNA expression in the blood and brain of rats subjected to transient focal ischemia by middle cerebral artery occlusion. Stroke 39: 959-966, 2008.

30. Chen X, Ba Y, Ma L, Cai X, Yin Y, Wang K, Guo J, Zhang Y, Chen J, Guo X, et al: Characterization of microRNAs in serum: A novel class of biomarkers for diagnosis of cancer and other diseases. Cell Res 18: 997-1006, 2008.

31. Selvamani A, Sathyan P, Miranda RC and Sohrabji F: An antagomir to microRNA Let7f promotes neuroprotection in an ischemic stroke model. PLoS One 7: e32662, 2012.

32. Cacialli P, Gueguen MM, Coumailleau P, D'Angelo L, Kah O, Lucini $\mathrm{C}$ and Pellegrini E: BDNF expression in larval and adult Zebrafish brain: Distribution and cell identification. PLoS One 11: e0158057, 2016.

33. Varendi K, Kumar A, Härma MA and Andressoo JO: miR-1, miR-10b, miR-155, and miR-191 are novel regulators of BDNF. Cell Mol Life Sci 71: 4443-4456, 2014.

34. Cao X, Pfaff SL and Gage FH: A functional study of miR-124 in the developing neural tube. Genes Dev 21: 531-536, 2007.

35. Budde H, Schmitt S, Fitzner D, Opitz L, Salinas-Riester G and Simons M: Control of oligodendroglial cell number by the miR-17-92 cluster. Development 137: 2127-2132, 2010.

36. Olive V, Bennett MJ, Walker JC, Ma C, Jiang I, Cordon-Cardo C, Li QJ, Lowe SW, Hannon GJ and He L: miR-19 is a key oncogenic component of mir-17-92. Genes Dev 23: 2839-2849, 2009. 